\title{
Piloter dans une société en changement
}

\section{Monica Gather Thurler}

Dans la plupart des systèmes scolaires, le pilotage revient aux cadres scolaires (collaborateurs des directions générales, chefs d'établissements, directeurs des institutions de formation des enseignants), qui sont censés exercer un leadership déterminant dans le développement de ces systèmes. En même temps, le raisonnement sur leur rôle et fonction reste encore trop souvent limité à quelques principes généraux, reliés à leur position dans une structure hiérarchique et à leur travail prescrit dans les cahiers de charge (lorsque ceux-ci existent), n'est que rarement fondé sur une analyse approfondie de leur travail réel. Or, d'un pays à l'autre, voire d'une région à l'autre dans les pays fédéralistes, celui-ci differe selon les législations et les orientations politiques, mais également selon la culture professionnelle prépondérante. De fait, leur manière d'exercer un leadership revêtira des fonctionnements et des formes très divers: ils se trouveront tantôt dans la position d'un décideur solitaire, dans celle d'un membre d'une équipe de direction ou encore dans celle d'un primus inter pares au sein d'une équipe pédagogique. A ces variations s'ajoutent un certain nombre d'aspects dont on ne mesure pas toujours les conséquences: la plupart des cadres scolaires ont au préalable été enseignants eux-mêmes, ce qui implique une certaine "complicité» avec celles et ceux qu'ils sont désormais censés diriger. Ils succèdent toujours à quelqu'un et, à un certain moment, cèdent leur place à quelqu'un d'autre; ils seront donc dans l'obligation d'assurer une certaine continuité tout en démontrant leur capacité de laisser leur "empreinte» pendant leur passage. Enfin, ils représentent l'autorité, qui leur délègue du pouvoir; ils doivent donc "montrer patte blanche", se soumettre à une culture institutionnelle, à une conception du rôle du ministère, des autorités politiques locales ou régionales, dont le rôle et l'influence varient d'un pays et d'un système scolaire à l'autre.

Pour lancer le débat sur la problématique du leadership qui se trouve au centre de ce congrès de la SSRE, je prendrai le parti de lier la fonction des cadres scolaires soit au fonctionnement ordonné du système éducatif, soit à sa modernisation continue. Et tenterai de montrer qu'en vertu de l'écart de moins en moins acceptable entre les objectifs déclarés de l'école et ses résultats, la composante de l'innovation semble ici prioritaire. Dans cette perspective, diriger dans une société en changement, c'est piloter des 
transformations incessantes, qu'elles soient impulsées par l'évolution de la demande sociale, les inflexions des politiques de l'éducation, le renouvellement des savoirs, voire les exigences des nouveaux publics scolaires, culturelles et économiques.

Dans la plupart des systèmes scolaires, le pilotage revient aux cadres scolaires (responsables politiques, collaborateurs des directions générales, chefs d'établissements, directeurs des institutions de formation des enseignants). Ils sont appelés à exercer un leadership déterminant et efficace, qui sera toujours l'aboutissement précaire de la recherche d'un fragile équilibre, entre maintien des acquis et mise en œuvre de changements nécessaires. Face à ces exigences, la fonction et les rôles des métiers de l'encadrement se définissent encore trop souvent à travers quelques principes généraux: un vague cahier des charges (lorsque celui-ci existe), une indication assez générale, concernant leur position dans la structure hiérarchique du système, la description d'un éventail de tâches et d'activités censées cerner le métier, voire un référentiel de formation plus proche du sens commun que d'une exploration systématique de leur travail réel.

Il en résulte une certaine centration sur la dimension avant tout administrative et gestionnaire de leur métier, qui ne parvient pas à tenir compte du fait qu'il differe d'un pays à l'autre, voire d'une région à l'autre dans les pays fédéralistes, selon les législations et les orientations politiques, mais également selon les cultures professionnelles respectives. Avec la conséquence que les cadres scolaires seront amenés à exercer, sous des appellations semblables, des fonctions plutôt diverses: ils se trouveront tantôt dans la position d'un décideur solitaire, dans celle d'un membre d'un conseil de direction ou encore dans celle d'un primus inter pares au sein d'une équipe pédagogique. À ces variations s'ajoutent un certain nombre de caractéristiques du métier dont il reste encore à clairement saisir les conséquences:

- Premièrement, la plupart des cadres scolaires ont au préalable été enseignants eux-mêmes, ce qui implique une certaine "complicité» avec les anciens collègues qu'ils sont désormais censés diriger. Il leur est difficile de s'en distancier sans être perçus, soit comme de "petits chefs", soit comme des collègues proches, incapables d'instaurer la distance et l'asymétrie indispensables pour piloter les processus de transformation en cours et à venir.

- Deuxièmement, leur marge de pouvoir (assurer le maintien de la qualité de l'existant tout en veillant à la transformation des pratiques de manière à la développer encore davantage) reste limitée dans la mesure où l'efficience de leur action dépendra de ce que les enseignants penseront et feront des prescriptions qui leur sont adressées. Ne pouvant être omniprésents pour contrôler la pertinence et qualité de leur mise en oeuvre dans les détails, leur éfficacité dépendra donc de leur capacité de construire un «leadership distribué» et de la volonté des enseignants de l'assumer.

- Troisièmement, ils succéderont toujours à quelqu'un et devront, à un certain moment, céder leur place à quelqu'un d'autre; ils seront donc dans l'obliga- 
tion d'associer continuité et rupture, en introduisant des changements sans pour autant anéantir le travail déjà effectué, en esquissant des projets d'avenir sans verrouiller le système, de manière à créer les bases d'un changement «durable», qui se poursuivra au-delà de leur départ.

- Enfin, les cadres scolaires représentent l'autorité, qui leur délègue du pouvoir. Ils doivent donc «montrer patte blanche», s'inscrire dans - et certaines fois se soumettre à - la culture institutionnelle qu'ils sont censés incorporer. À l'heure de la mondialisation des échanges humains et du pilotage toujours plus globalisé des systèmes éducatifs, ils sont en outre appelés à jouer la courroie de transmission qui relie le ministère et les autorités locales ou régionales aux opérateurs du terrain, dans un contexte politique et social de changements multiples et permanents.

Autrement dit: dans un contexte qui valorise désormais la décentralisation et l'autonomie partielle des instances locales, les cadres sont encore davantage que dans le passé responsables de leur fonctionnement coordonné et cohérent. Il leur appartient désormais de piloter un système éducatif chargé de se transformer et de développer la qualité dans la durée. Dans cette perspective, il importe qu'ils sachent identifier les contraintes contradictoires auxquelles se trouvent confrontés les acteurs du terrain appellés à honorer les standards définis par le système; qu'ils soient prêts à explorer de nouvelles dimensions de l'organisation du travail permettant de dépasser, à terme, la traditionnelle gestion des ressources humaines, du temps et des espaces, et des dispositifs de l'enseignement-apprentissage s'étant avérés d'une efficacité limitée. Enfin, il leur appartiendra d'introduire de nouveaux modes d'évaluation, mieux à même d'assurer l'efficacité de l'action collective.

Avec Perrenoud (1998), je propose de revenir à la question suivante: les périodes de crise et de transformation changent-elles, oui ou non, les métiers de l'encadrement et, par conséquent, les besoins en matière de leadership ? Et d'y répondre en affirmant que ces périodes accentuent sans doute certaines de leur caractéristiques ordinaires, en dévoilant en même temps les facettes intimes du travail des acteurs concernés: l'incertitude, le conflit, les contradictions entre les fins et les moyens, la diversité des attentes et des logiques d'action des membres de l'organisation aussi bien que des usagers; la disparité des situations, des charges, des compétences et des volontés d'engagement des collaborateurs, avec les problèmes de justice qui s'ensuivent; la gestion au quotidien de la tension entre le désir de cohérence et la nécessité de passer des compromis, les nobles causes et la défense des intérêts acquis, la politique à long terme et les urgences du quotidien; enfin, faire la part entre l'identification à un sous-système et le souci de l'ensemble.

Dans cette perspective, piloter dans une société en changement - et au niveau du système ou sous-système dont on est responsable - c'est certes assurer et donc contrôler le maintien de la qualité acquise. Mais c'est aussi contribuer au développement dans la durée, en lien avec l'évolution constante de la demande so- 
ciale, le renouvellement des savoirs, les réalités culturelles et économiques, enfin, les inflexions données par les politiques de l'éducation. Piloter, c'est relever, au quotidien, les innombrables défis qui découlent de la tension existante entre centration sur les résultats et centration sur le processus, entre exigences du système et exigences du local. Piloter, c'est, enfin, apprendre à identifier et affronter les contradictions et injonctions paradoxales émanant des prescripteurs, gérer les ambivalences et crises identitaires de ceux qui sont censés mettre en œuvre les décisions de leur hiérarchie, enfin, de faire la part entre l'obligation d'assurrer la cohérence du système et la nécessité de prendre en compte les marges d'action des acteurs locaux.

La littérature sur le changement dans les systèmes éducatifs (Alter, 2002; Bronckart \& Gather Thurler, 2004; Perrenoud, 2005) ne cesse de rappeler qu'aucune réforme n'est «magique», qu'aucune restructuration n'aboutit "automatiquement» à des améliorations. Tout dépendra d'un savant mélange entre logiques d'acteurs et stratégies de management, qui parvient à articuler sans relâche les activités pédagogiques entre elles, et à tenir compte des priorités des acteurs locaux sans pour autant nier les finalités déclarées du système. Il semblerait que cette responsabilité d'articulation représente l'un des aspects essentiels du leadership transformationnel que sont aujourd'hui censés exercer les personnels de l'encadrement.

\section{Leadership: risques de distorsion Linguistique}

D’origine anglo-saxonne, ce terme est difficile à définir de façon univoque, d'autant plus que la littérature - scientifique et popularisante - multiplie ses modes d'utilisation. Depuis une vingtaine d'années, il est associé aux nouvelles approches de gouvernance des systèmes publiques et éducatifs, qui sont allés emprunter leurs modèles de gestion au monde des affaires et du management. Pour Starrat (2001), ce phénomène des «isomorphismes culturels» favorise l'intégration dans le langage courant de concepts apparemment semblables et convaincants, alors qu'ils sont en réalité fondés sur des présupposés et des contextes socio-éducatifs et politiques différents. Dans la mesure où ces concepts sont diffusés à partir de publications anglophones, celles et ceux qui s'en inspirent dans d'autres régions linguistiques sont finalement contraints à non seulement adopter les vocabulaires pour la plupart «intraductibles», mais également les idéologies managériales et organisationnelles qu'elles véhiculent. Leur mise en œuvre, souvent trop rapide (car soucieuse de produire des effets à court terme) et trop autoritaire (car elle se heurtera inévitablement aux résistances de celles et ceux qui ont le sentiment qu'elles ne prennent guère en compte leur travail réel), se heurtera inévitablement aux habitudes langagières et comportementales des acteurs concernés, qui se sont forgées dans une réalité contextuelle, culturelle et sémantique différente. 
Il est fort probable que le concept leadership n'échappe pas à cette règle. L'un des facteurs majeurs du risque de distorsion réside notamment dans la connotation fortement participative que lui accordent certains auteurs (Hargreaves \& Fink, 2006; Spillane, 2006), en l'articulant au processus de professionnalisation des métiers de l'éducation. Alors que sa transposition aux contextes politiques et sociaux dont la dimension historique et évolutive reste encore fortement marquée par des logiques centralisatrices et bureaucratiques, risque de le réduire à sa plus simple expression de gestion et de contrôle.

\section{Dilemmes, contradictions et injonctions parado- xales dans les sociétés modernes}

L'une des particularités des sociétés modernes consiste ainsi, selon Moos \& Møller (2003), à prendre en compte la complexité des entités «lâchement couplées» et difficilement contrôlables que représentent les systèmes scolaires.Dans cette perspective, deux logiques d'action - en partie contradictoires - interagissent afin de relever les défis de notre société postmoderne: d'une part, il s'agit de relâcher le contrôle pour accorder une certaine autonomie aux acteurs, appelés à développer des solutions plus efficaces pour résoudre les nombreux problèmes du local; d'autre part, il est urgent d'instaurer de nouvelles mesures de contrôle, d'abord pour rétablir la confiance des usagers et ensuite pour générer des savoirs de pilotage (Strittmatter, 2004). Ces deux logiques d'action - en apparence contradictoires - viennent renforcer les tensions conflictuelles du système: hiérarchie versus communauté apprenante; contractualisation versus réciprocité; soumission versus construction de sens; standardisation versus logique d'action locale...

Les cadres du système scolaire - tout autant que les enseignants - devront vivre avec les contradictions et paradoxes. D'une part les nouvelles politiques de gestion avec leur management top-down et leur renforcement de systèmes de contrôle; d'autre part l'analyse sociologique qui montre que les sociétés complexes résistent aux entreprises managériales, car la tendance générale oriente vers l'innovation et l'avenir, au lieu de rester «collé» au passé ou le présent. Il reste donc à développer la confiance - et le plaisir -, indispensables pour oser être acteur dans un monde menacé par l'irrationalité et l'incertitude (Starratt, 1993, trad. Gather Thurler).

Sergiovanni (2004)) insiste à ce propos sur la nécessité d'établir des communautés scolaires cultivant un leadership partagé: «dans de telles communautés, le leadership, qui se limite habituellement au pouvoir sur les événements et sur les collaborateurs est ainsi redéfini en tant que pouvoir investi dans la réalisation d'objectifs communs» (p. 179, visant à renforcer l'empowerment de l'ensemble des acteurs concernés.

En dépit des efforts investis dans la professionnalisation des métiers de l'éducation, dans le but de permettre aux membres du corps enseignant d'accéder à 
davantage de pouvoir d'autodécision et d'autodétermination, il n'est cependant pas certain que les cultures scolaires actuelles permettent cette évolution, qui représente une rupture nette avec l'habitude d'attribuer les dysfonctionnements à des tiers: l'hiérarchie, les collègues, les parents, les élèves... Argyris (1998), dans un article intitulé Les nouveaux vêtements de l'empereur, montre que l'empowerment est généralement une mission impossible dans des organisations qui sont fortement marquées par les prescriptions et les habitudes de contrôle. Les travailleurs y résistent presque systématiquement à l'idée d'un empowerment, soit parce qu'ils n'y croient tout simplement pas, soit parce qu'ils ne sont guère disposés à offrir la contrepartie exigée: leur engagement soutenu et durable dans une exploration de leurs moyens d'action et marges d'autonomie, permettant de rendre plus efficace l'action pédagogique; en même temps, un rendre compte non seulement des avancées réalisées, mais également des difficultés rencontrées.

Argyris (ibid.) insiste ainsi sur le fait que dans certains systèmes, fortement fermés durant de nombreuses années, l'empowerment et le leadership distribué représentent une charge supplémentaire que les enseignants ne sont pas prêts à assumer. Il s'avère en outre que les cadres de l'éducation manifestent eux-mêmes une certaine réticence à développer l'empowerment de leurs collaborateurs. Car ils ont fait l'expérience que le partage du pouvoir n'est jamais totalement assumé en présence de situations difficiles; en même temps, ils ont appris - certaines fois à leur dépens que l'empowerment - pourtant révendiqué et bien vécu au début - ne résiste guère au surcroît obectif de travail, ni à l'usure du quotidien. Une fois la première excitation passée, les enseignants préferent revenir au status ante quo.

\section{Développer le leadership, c'est développer la recherche}

Transformer le système éducatif est une action complexe. Cela exige des savoirs d'action, des compétences, des savoirs déclaratifs ou procéduraux. Lesquels ? Il serait sans doute d'un grand apport si les futures recherches tentaient de mieux décrire le travail réel des cadres de l'éducation, d'identifier les savoirs d'action et d'innovation qu'ils mobilisent pour transformer les pratiques et les structures au sein des lieux de formation dans lesqeuls ils interviennent. Une partie du travail typologique pourrait ainsi porter sur les objets de ces savoirs: les uns visent les organisations, d'autres le champ politique, d'autres les micro-interactions ou les processus intrapersonnels.

Certains savoirs sont plutôt stratégiques ou tactiques, ils conduisent à créer des alliances, des événements, des mouvements favorables à l'innovation. D'autres sont plutôt argumentatif ou persuasifs, ils visent à convaincre, à faire évoluer les représentations. Indépendamment de cette liste plutôt «intuitive», j’estime qu'il existe trois raisons d'investir la problématique du leadership par la recherche: 
- sur le plan épistémologique, le rapport aux pratiques sociales et du fonctionnement des institutions sont un moteur de développement de la recherche dans sa globalité, non seulement de la recherche appliquée ;

- pour des raisons politico-stratégiques, il est important que la recherche se positionne face à l'émergence massive du New Public Management dans les politiques de Gouvernance, qu'elle se positionne, ouvre le débat, documente les contradictions, paradoxes et impasses, et, si possible, propose des alternatives;

- enfin, pour des raisons culturelles, il est fortement indiqué que l'évolution du pilotage des systèmes éducatifs reste connecté à la recherche, à la pensée critique et à une certaine autonomie intellectuelle.

\section{Bibliogaphie}

Alter, N. (Ed.) (2002). Les logiques de linnovation - Approche pluridisciplinaire. Paris: La Découverte.

Argyris, C, (1998). Les nouveaux vêtements de l'empereur, Harvard Business Review, MayJune, 98-105.

Bronckart, J.-P. \& Gather Thurler (Ed.) (2004). Transformer l'école. Bruxelles: De Boeck, Coll. Raisons Educatives.

Hargreaves, A. \& Fink, D. (2006). Sustainable leadership. San Francisco: Jossey-Bass.

Moos, L. \& Møller, J. (2003). Schools and Leadership in Transition: the case of Scandinavia, Cambridge Journal of Education (2003), 33 (3), 353-371.

Perrenoud, Ph. (1998). Diriger en période de transformation ou de crise, n'est-ce pas, tout simplement, diriger ? In G. Pelletier \& R. Charron (Ed.), Diriger en période de transformation (pp. 7-30). Montréal: Éditions AFIDES.

Sergiovanni, T. J. (2004). Strengthening the Heartbeat: Leading and Learning Together in Schools.

Spillane, J.sP. (2006). Distributed Leadership. Jossey-Bass: San Francisco.

Starratt, R.J. (1993). The Drama of Leadership. London, Washington DC: Falmer Press.

Strittmatter, A. (2004). Jusqu'où peut-on et doit-on standardiser l'École ? In J.-P. Bronckart, J.-P. et Gather Thurler, M. (dir.) Transformer l'école (pp. 193-217). Bruxelles: De Boeck, Coll. Raisons Educatives,

Mots clés: Leadership, analyse de la profession, objectifs, innovations.

\section{Führen in einer Gesellschaft in Veränderung}

\section{Zusammenfassung}

In den meisten Schulsystemen wird die Steuerung von Schule in das schulische Umfeld verlegt: Mitarbeitende der Bildungsverwaltung, Schulleiter, Direktoren von Ausbildungsinstitutionen sollen künftig vermehrt die Qualitätsentwiclung (mit)steuern. Allerdings bleibt die Reflexion ihrer Rolle und Funktion zu oft begrenzt auf einige allgemeine Prinzipien, die durch ihre Position in einer hierarchischen Struktur ihre durch Pflichtenhefte (wenn diese existieren) vorgeschriebene Aufgabenbereiche bestimmt sind und auf ihre Arbeit, anstatt sich auf eine 
vertiefte Analyse ihrer tatsächlichen Arbeit einzulassen. Beim Blick von einem Land zum anderen, in den föderalistischen Ländern von einer Region zur anderen, findet man Unterschiede zwischen den Gesetzgebungen und politischen Orientierungen, aber auch zwischen der vorherrschenden professionellen Kultur.

In Tat und Wahrheit üben SchulleiterInnen sehr untrschiedliche Formen und Funktionen von Leadership aus: Man findet sie in der Position eines einsamen Entscheiders, als Schulleitungsmitglied oder als primus inter pares einer pädagogischen Gruppe. Dazu kommen einige weitere Aspekte, deren Konsequenzen nicht immer berücksichtigt werden: Die meisten Schulleiter waren vorher ihrerseits Lehrpersonen: sie müssen als Vorgesetzte von früheren Kollegen auftreten, mit denen sie im Prinzip ein gutes Einverständnis entwickelt haben. mit ihnen impliziert und welches sie fortführen müssen. Schulleiter übernehmen immer die Funktion eines Vorgängers und geben diese zu einem bestimmten Zeitpunkt wieder an eine andere Person ab. Sie haben daher die Verpflichtung, eine gewisse Kontinuität zu sichern und gleichzeitig ihre Fähigkeit zu zeigen, während ihrer Amtszeit ihren persönlichen Stempel aufzudrücken. Zudem verkörpern sie die Autorität, welche ihnen Macht delegiert. Sie müssen sich also einer institutionellen Kultur unterwerfen, einer Rollenkonzepten des Ministeriums und lokaler sowie regionaler politischer Autoritäten bzw. Institutionen, deren Bedeutung und Einfluss sich zwischen Ländern und Schulsystemen unterscheidet.

Um die Diskussion über die Leadershipproblematik zu lancieren, die das Kernthema des Kongresses der SGBF bildet, werde ich die Funktion der Schulleiter im Hinblick auf die Funktionsweise und fortlaufende Modernisierung des Erziehungssystems untersuchen. Ich werde zeigen, dass wegen immer weniger akzeptablen Unterschieden zwischen den Zielen und Wirkungen der Schule, die Innovation als Ganze prioritär wird. In der Perspektive einer ständig sich verändernden Gesellschaft sind Schulleitungen folglich aufgefordert, kontinuierlich ablaufende Transformationen zu steuern, unabhängig davon, ob diese durch veränderte gesellschaftliche Wertvorstellungen, Umorientierungen der Bildungspolitik, neue Wissensbestände, bzw. neue Anfordungen der Schülerpopulationen ausgelöst werden

Schlagworte: Leadership, Berufsanalyse, Ziele, Schulentwicklung Veränderungsprozesse, Innovation

\section{Pilotare in una società in cambiamento.}

\section{Riassunto}

Nella gran parte dei sistemi scolastici, il pilotaggio è una mansione rivestita dai quadri scolastici (collaboratori di direzioni generali, capi d'istituti, direttori di istituti di formazione degli insegnanti), che dovrebbero esercitare una leadership determinante nello sviluppo di questi sistemi. Al tempo stesso, la reflessione sul 
loro ruolo e la loro funzione resta ancora troppo spesso limitata a qualche principio generale, relativo alla loro posizione in una struttura gerarchica e al loro lavoro previsto nei contratti, invece di concentrarsi su analisi approfondite del loro lavoro concreto. Ora, da un paese all'altro o da una regione all'altra nei paesi federalisti, questo lavoro differisce in funzione delle legislazioni e dagli orientamenti politici, ma anche in funzione della cultura professionale preponderante. Infatti, la loro leadership rivestirà dei funzionamenti e delle forme molto differenti: si troveranno a volte nella posizione di un dirigente solitario, in quella di un membro di un'équipe di direzione o ancora in quella di un primus inter pares in seno all'équipe pedagogica. A queste variazioni si aggiungono una serie di aspetti dei quali non riesce sempre a misurare le implicazioni: la maggior parte dei quadri scolastici sono stati precedentemente degli insegnanti. Il che implica una certa "complicità» con coloro che oramai devono dirigere. Inoltre essi succedono sempre a qualcuno e, a un certo punto, lasciano il loro posto a qualcun altro. Questo li mette nell'obbligo di assicurare una certa continuità, pur potendo dimostrare la propria capacità di lasciare una "traccia» durante il loro passaggio. Infine, rappresentando l'autorità, che delega loro potere, essidevono sottomettersi a una cultura istituzionale, a una concezione di questo ruolo che ha il dipartimento competente, alle politiche locali o regionali, istituzioni il cui ruolo e l'influenza variano da un paese e da un sistema scolastico all'altro.

Per lanciare il dibattito sulla problematica della leadership che si trova al centro di questo congresso della SSRE, cercherò di legare la funzione dei quadri scolastici sia al funzionamento ordinato del sistema educativo sia al continuo processo di modernizzazione. Tenterò di mostrare come, in virtù dello scarto sempre meno accettabile tra obiettivi dichiarati della scuola e i suoi risultati, la componente dell'innovazione sembra qui prioritaria. In questa prospettiva, dirigere in una società in mutamento, significa pilotare delle trasformazioni incessanti, stimolate dall'evoluzione della domanda sociale, dalle inflessioni delle politiche dell'educazione, dal rinnovo dei saperi o dalle esigenze dei nuovi pubblici scolastici, culturali e economici.

Parole chiave: Leadership, analisi della professione, obiettivi, sviluppo scolastico, processi de cambiamento, innovazione

\section{Piloting in a changing society}

Summary

In most school systems piloting belongs to school officials (executive boards, principals, directors of institutions that train teachers), who are supposed to show leadership in the development of these systems. At the same time, the reasoning on their role and function still remains too often limited to some general principles, linked to their position within a hierarchical structure and to their job within a mandate (when it exists), instead of being founded on a thorough 
analysis of their real work. Now, from one country to another, or from one region to another in federalist countries, their work will varie according to the different legislations and political orientations, but also according to the predominant professional cultures: will thus schoolleaders take on very diverse functionings and forms : they will be either lonely decision makers, or members of an executive board, or a primus inter pares in a pedagogical team. To these variants one must add a number of aspects whose consequences are not always measured: most school officials have already been teachers themselves, thus leading to some sort of "complicity» with those they are supposed to steer. They always come after someone and they are supposed to leave their place to their successors at some point; they will then have to guarantee continuity while trying to leave a mark during their stay. Finally, they represent the authority that empowers them; they thus have to abide by an institutional culture, a conception of the role of the ministry, of local or regional authorities, institutions whose role and influence change from a country to another and from a school system to another.

To kickstart the debate on the issue of leadership that lies at the center of this SSRE congress, I will try to link the function of school officials either to the proper functioning of the school system, or to its continuing modernisation. I shall also try to show that, given the less and less bearable gap between the declared objectives of the school and its achievements, innovations seem a priority. From this point of view, steering in a changing society means piloting continuing transformations, be they the result of the evolution of social demands, of changes in education policy, of the evolution of knowledge, or of the needs of new school, cultural and economic audiences.

Keywords: Leadership, profession analysis, objectives, school developement, change processes, innovation 